\title{
Perception of the importance of oral hygiene in a group of adults from South-West Romania
}

\author{
Dora Maria Popescu', Andrada Soanca², Allma Pitru³, Alex Salan, \\ Dorin Nicolae Gheorghe' ${ }^{1}$, Petra Surlin ${ }^{1}$ \\ ${ }^{1}$ Department of Periodontology, Craiova University of Medicine and Pharmacy, Craiova, Romania \\ 2 Department of Periodontology, "Iuliu Hatieganu“ University of Medicine and Pharmacy, Cluj-Napoca, Romania \\ ${ }^{3}$ Department of Oral Pathology, Craiova University of Medicine and Pharmacy, Craiova, Romania \\ ${ }^{4}$ Department of Oral Surgery, Craiova University of Medicine and Pharmacy, Craiova, Romania
}

Oral hygiene is a dental procedure with the role of removing bacterial plaque and action to prevent oral diseases, including periodontal disease. Oral prophylaxis includes both daily dental hygiene (daily brushing, flossing and mouthwash) and the visits to the dentist for professional hygiene and prophylaxis.The correct brushing of the teeth is important not only for maintaining oral health but also for the health of the whole body, because there are correlations between diseases of the oral cavity and problems of the digestive tract or even heart disease.

Keywords: oral health, oral hygiene, toothbrush

\section{INTRODUCTION}

Oral health contributes to the overall health of the body and to maintaining the physiological balance of many organs and can often be associated with their health.Poor oral hygiene can cause tooth decay, gum disease and has also been associated with heart disease, cancer and diabetes [1].

Oral hygiene is a daily activity that has the role of keeping the teeth and oral cavity clean to prevent the occurrence of tooth decay or periodontal disease. Cleaning of the teeth and oral cavity has become today a priority and should be performed whether the teeth are natural or artificial, crowns or dental bridges, implants or removable dentures. If in the past, the focus was on dental hygiene to prevent dental diseases, especially tooth decay, today, it is considered that the use of dental hygiene products also helps to prevent or alleviate diseases of the soft tissue in the oral cavity [2].
According to the World Health Organization: 60$90 \%$ of students have at least one decayed tooth; almost $100 \%$ of adults have at least one decayed tooth; almost $20 \%$ of adults between the ages of 35 and 44 suffer from severe gum disease [3]; about $30 \%$ of people between the ages of 65 and 74 no longer have natural teeth; in most countries, there are between 1 and 10 cases of oral cancer per 100,000 people [4].

According to statistics, the incidence of periodontal disease has increased in recent years, and has reached an alarming percentage, becoming the "disease of the century" in dentistry. Thus, about $70 \%$ of the world's population suffers of periodontal disease, either in mild forms manifested by simple gingival bleeding diagnosed as gingivitis, or in severe forms such as periodontitis manifested by progressive loss of all teeth.

The basis of dental hygiene is dental care which every patient performs at home, by brushing their 
teeth after every meal. To this method can be added auxiliary procedure such as: the use of interdental floss, mouthwash, mouth irrigation etc. [5].

These additional brushing methods are necessary to clean the interproximal surfaces of the teeth as effectively as the facial, lingual and occlusal surfaces.These simple and effective methods aim to reduce the quality and quantity of biofilm accumulation, the cause of periodontal inflammation.

Daily dental hygiene prevents gingivitis, which occurs with periodontal disease and helps to detect the symptoms of systemic diseases that may manifest in the oral cavity [6].

In addition to poor hygiene, there are many local and general factors that can increase the risk of periodontal disease development and progression by acting as plaque retention factors. Local factors that it could be mentioned are: calculus, carious lesions, malocclusion, inappropriate prosthetic, edentations, iatrogenic factors, orthodontic therapy, smoking. In addition, a number of systemic conditions can affect the state of oral health and, consequently, periodontal health. Over the years, a large number of studies have associated oral disease to many systemic conditions. These include cardiovascular disease, diabetes, adverse pregnancy outcomes, obesity, liver disease or neurodegenerative diseases [7].

\section{AIM}

The aim of the study is to assess the perception of young adults of the importance of oral hygiene and all related procedures. We aimed to evaluate the behavior and trends of modern people from different backgrounds regarding oral hygiene in order to have a more adequate perspective on the prevalence of oral health problems caused by poor hygiene.

\section{MATERIAL AND METHOD}

We collected the data required for the study using a questionnaire obtained from patients presented between February and April 2019 in the Department of Periodontology of University of Medicine and Pharmacy from Craiova, Romania. The 70 patients included in that study had to be aged between 20 and 40 years old, from both rural and urban areas. The questionnaire was approved by the Ethics Committee of the University of Medicine and Pharmacy of Craiova. The questions were formulated in such a way that as much information relevant to the subject as possible could be collected and the attitudes of those concerned could be easily as certained. The data was analyzed with Microsoft Excel programme (Microsoft Inc, Redmond, WA, USA).

We tried to reach all the important points to find out how the young adults who participated in the study practice their oral hygiene and we included in the study 10 questions relevant to the topic, questions from which conclusions could be drawn about the subjects' attitude towards dental health (Table 1).

\section{RESULTS}

The study was conducted with a number of 70 participants, out of which 37 were males and 33 were females. Of the 70 participants in the study, 12 were between 20 and 24 years of age (17.14\%), 25 were between 25 and 29 years of age (35.71\%), 16 were between 30 and 34 years of age (22.86\%), and 17 were between 25 and 40 years of age(24.29\%). All participants fall into the young adults group as they are between 20 and 40 years old.

In order to collect the most significant data on attitude towards oral hygiene, we selected participants from both rural and urban areas: 27 of the participants (38.57\%) were from rural areas, while the other 43 (61.43\%) were from urban areas. There is a positive difference in dental health maintenance among the participants from urban area.

The level of education of the individual often has an impact on education in relation to oral health care. For the study, we interviewed individuals whose last study was either academic degree or high school, more specifically 52 of the subjects $(74.29 \%)$ had academic degree, while 18 of them (25.71\%) have completed high school.

TABLE 1. Questions from the questionnaire

\begin{tabular}{|c|c|c|c|c|c|}
\hline Patient information & Name & Gender & Age & $\begin{array}{l}\text { Rural / Urban } \\
\text { environment }\end{array}$ & Completed studies \\
\hline \multicolumn{6}{|c|}{ Question: } \\
\hline \multicolumn{3}{|c|}{ 1. How often do you go to the dentist? } & \multicolumn{3}{|c|}{ 6. What kind of toothbrush do you use? Manual or electric? } \\
\hline \multicolumn{3}{|c|}{ 2. What do you expect during your visit to the dentist? } & \multicolumn{3}{|c|}{$\begin{array}{l}\text { 7. What kind of toothpaste do you use?Example: whitening } \\
\text { effect, sensitive teeth, gingival bleeding, against bad breath. }\end{array}$} \\
\hline \multicolumn{3}{|c|}{ 3. How often do you brush your teeth? } & \multicolumn{3}{|c|}{ 8. Do you use other additional methods of dental hygiene? } \\
\hline \multicolumn{3}{|c|}{ 4. How much time do you spend brushing your teeth? } & \multicolumn{3}{|c|}{$\begin{array}{l}\text { 9. When's the last time you had your teeth professionally } \\
\text { cleaned? }\end{array}$} \\
\hline \multicolumn{3}{|c|}{ 5. How often do you change your toothbrush? } & \multicolumn{3}{|c|}{$\begin{array}{l}\text { 10. Are you a smoker? If so, for how long? How many cigarette } \\
\text { do you smoke daily? }\end{array}$} \\
\hline
\end{tabular}


The importance of oral hygiene of each study participant can be easily seen from the number of actions (consultations, visits etc.) in a dental clinic. $30 \%$ of the study participants responded that they visit a dentist once a year, $48.57 \%$ responded that they visit a dentist twice a year, and 21.43 responded that they only go to the dental clinic only when they have pain.

From the questionnaire, we found that $21.43 \%$ of the respondents brush their teeth only once a day, and the remaining, $78.57 \%$ brush their teeth twice a day.

Out of the 70 respondents, 17 (24.29\%) said that they brush their teeth for 30 seconds, 23 (32.86) said that they spend 1 minute brushing their teeth, and 30 (42.86) said that they spend at least 2-3 minutes on dental hygiene.

When asked about toothbrush replacement, 40\% of the participants answered that they change toothbrush every 3 months, 34.29\% change toothbrush twice a year and $25.71 \%$ change it only once a year.

Toothbrushes can be electric or manual, among the participants, 28 use electric toothbrush and 42 people use manual toothbrush.

The toothpaste used must be selected according to each individual's needs, existing conditions, preferences, medical recommendations etc. Of the group included in the study, 16 subjects (22.86\%) reportedusing toothpaste for sensitive teeth, $20 \mathrm{sub}$ jects $(28.57 \%)$ use whitening toothpaste, 16 participants $(22.86 \%)$ use bleeding gums toothpaste, and 18 of them (25.71\%) use bad breath toothpaste.

Oral hygiene is not only about brushing teeth, there are many additional methods of oral hygiene. Among the subjects, $20 \%$ use dental floss, $35.71 \%$ use mouthwash, $18.57 \%$ use interdental brushes, and $25.71 \%$ do not use any oral hygiene methods other than brushing their teeth.

Professional teeth scaling or cleaning is important because plaque and calculushave a harder time depositing on a smooth, clean tooth surface. A professional procedure such as ultrasonic scaling, performed by a dentist, is a key part of proper oral hygiene and plays an essential role in preventing dental diseases such as gingivitis. Based on the questionnaire we found that $38.57 \%$ of the subjects have performed the last professional dental hygiene procedure estimated 3 months ago, $10 \%$ of the subjects have performed the procedure about 6 months ago, $34.29 \%$ of them have performed the procedure one year ago, while $17.14 \%$ of the participants answered that they have never performed this dental hygiene procedure.

In the questionnaire, subjects were asked if they smoked or had smoked in the past, and if they answered in the affirmative, they were asked how many cigarettes they smoked per day.The questionnaire asked subjects if they smoked or had smoked in the past, and if their answer was yes, they were asked how many cigarettes they smoked per day. 26 study participants answered yes to the question of whether they smoked or had smoked, and 44 answered that they had never smoked.Regarding the number of cigarettes smoked daily, $46.15 \%$ of the subjects smoked / smoked less than 6 cigarettes per day, $23.08 \%$ smoked / smoked one pack per day and $30.77 \%$ smoked / smoked 2 packs per day.

\section{DISCUSSION}

Following the study, we found differences in young adults' attitudes towards oral hygiene depending on a number of factors including the subjects' environment of origin and their most recent degree.

Although the discrepancy was not large, better attitudes were observed among subjects from urban environments who had completed faculty as their most recent degree that amongsubjects from rural areas who had completed high school as their most recent degree. The greater importance given to oral hygiene in urban areas is evident in this study, but the fact that the difference inrural subjects is not great is encouraging it shows an improvement in health education in less developed areas of the country. A study published in 2014 concluded that the majority of the Romanian population does not brush their teeth in the evening, but only in the morning, because they equate brushing with the moment of social interaction, and consider it an aesthetic act, rather than one for their own health. More than $45 \%$ of participants in the study reported that they brush their teeth less than twice a day, while $10 \%$ brush their teeth only a few times a week, once a week or occasionally.The same study, reported the use of toothpaste and toothbrushes in Romania, in 2013 and 2014. Thus, in 2014 on average, 1.5 tubes of toothpaste/year were used and a toothbrush for 1.5 years. The Health Behavior in School-Aged Children's Research Network, a global collaboration for transnational studies, collects data every four year on the health, social environment and behavior of children aged 11, 13 and 15. These years mark a period of increasing independence, which may influence the development of their health behaviors [8].

The Regional Public Health Center in Iasi conducted a study in 2016 and 2017, to determine students' knowledge, attitudes and behaviors related to oral health and dental hygiene as well as the main factors that influence oral health, including, among others, the child's eating habits and frequency of tooth brushing. In 2017, the study was conducted with 451 students from public schools in Iasi.The analysis of the responses showsthat $99 \%$ of the respondents consider it necessary to brush their 
teeth, thus acknowledging the importance of oral hygiene. About $74 \%$ of children brush their teeth at least twice a day. Most students (73\%) brush their teeth for at least 2 minutes, with a higher percentage among girls. More than half of the students change their toothbrush every three months. Regarding the frequency of visits to the dentist, $70 \%$ indicated that they only take action when dental damage occurs. Regarding eating habits that may affect oral health, most children in the lower grades indicated that they do not eat candy or sour juices between meals every day, but the behavior changes as children get olderand start eating candy once or twice, several times a day. Most students in grades 5-8 are confident that they know how to brush their teeth properly and that they have been trained in propertoothbrushing technique (79.3\%), with training provided mainly by the dentist (61.2\%). and parents (21.4\%). When choosing toothpaste, students focus more on toothpastes that whiten teeth, contain menthol and fluoride. In addition to toothbrush, students also use other methods of oral hygiene, such as mouthwash (61.3\%) and interdental brushes (4.4\%) [9].

In 2010, a study of the Directorate-General for Health and Consumers and coordinated by the Directorate-General for Communication, presenting data on the oral health of the population in the Member States of the European Union, published the following results:

- A small proportion of the European population (41\%) say they still have all their natural teeth. In Romania, the percentage is $30 \%$.

- Of those who no longer have all their natural teeth, almost a third (31\%) wear a removable prosthesis, with small differences from country to country. In Romania, $14 \%$ of respondents said they wear a removable prosthesis.

- Most Europeans say they have not experienced any difficulties or embarrassment about their teeth in the last twelve months. About $15 \%$ say they have difficulty chewing due to dental injuries; in Romania the percentage is $32 \%$. About $16 \%$ had pain in their teeth or gingival tissue. More than $7 \%$ felt embarrassed by the aesthetic appearance of their teeth; in Romania the percentage is $16 \%$ - the first place in Europe. Only $4 \%$ of Europeans have avoided conversation or limited their participation in social activities in the last twelve months due to oral health problems [10].

- On average, Europeans eat / drink 5 times a day. These are the moments when their teeth come into contact with food and sweet drinks, which can have an impact on oral health.
- A large majority of Europeans (88\%) believe that they can see a dentist within $30 \mathrm{~min}$ utes of their home or workplace if needed; in Romania the percentage is $81 \%$.

- They are also almost unanimous (92\%), in believing that they have access to a dental practise or clinic when needed.

- In Romania, the reasons for the last dental visit were routine check-up or cleaning treatments $(27 \%)$, routine treatments $(31 \%)$ and emergency treatments (40\%) [11].

Studies by NIDCR oral hygiene health and prevalence of dental caries among adults aged 20-64 years indicate a significant increase in dental caries and oral hygiene problems. However, it should be noted that this increase is caused by large discrepancies between certain groups of people, who live in poor areas or with low educations levels and have poor oral health, which affect the percentage of studies [12].

In the study conducted by HBSC, on children's oral health in Romania, the frequency of oral hygiene behavior of students was measured in one way: how often subjects brush their teeth.

The results of the study show that less than half of Romanian students brush their teeth at least twice a day (43.25\% of 11-year-old students, $46.55 \%$ of 13 -year-old students and $45.15 \%$ of 15 -year-old students).Among boys, it can be observed that the frequency of brushing teeth decreases with increasing age. For girls, the trend is the opposite, increasing. According to the reported results, every second student in Romania brushes their teeth no more than once a day. Regular and correct tooth brushing is a simple prerequisite for improving the oral health of children and adolescents [8].

According to a 2019 study on the level ofknowledge and skills of students regarding oral health most of the participants chose toothpaste with whitening effect, namely $35 \%$, and almost $25 \%$ considered the type of toothpaste rather insignificant [13].

In a study, emphasis was placed on the analysis of risk behaviors for oral health in order to identify the main problems that lead to pathological changes in oral health and statistics an adults' attitude towards oral hygiene practices were compiled. The national study shows that $2 \%$ of adults do not practice the minimum necessary oral hygiene. In addition, $10 \%$ of adults brush their teeth less than once a week [14].

Another data from a comparative study conducted on adults in three different countries states that only $55 \%$ of Romanian subjects reported still having 20 or more teeth.The statistical data show that the Romanian population has the greatest need for improvement in oral health [15]. Significant differences were also observed in dental clinic visits. The na- 
tional study shows that $18 \%$ of the country's population, children and adults, had no routine dental procedures or dental consultations at all in the last 5 years. Even more worrying is that $21 \%$ of them used the services offered only when they are in pain [14].

$48 \%$ o the participants in the study we conducted had a dental check-up every 6 months.

\section{CONCLUSIONS}

The fact that about $75 \%$ of the participants used alternative methods of oral hygiene in addition to daily tooth brushing shows that young adults al- ready tend to pay more attention to oral health and that there is an increased interest in this topic.The importance of the inflammatory response caused by the accumulation of biofilms in the oral cavity must be emphasized in discussions with patients. In addition to educating patients on proper tooth brushing technique and flossing, recommending a mouthrinse. Based on the results obtained by analyzing the questionnaires of the subjects who participated in this study, we support the idea that special attention should be paid to oral hygiene by improving the level of knowledge regarding these procedures.

Conflict of interest: none declared Financial support: none declared

\section{REFERENCES}

1. Fiorillo L. Oral Health: The First Step to Well-Being. Medicina (Kaunas). 2019 Oct 7;55(10):676.

2. Yamashita Y, Takeshita T. The oral microbiome and human health. J Oral Sci. 2017; 59(2):201-206.

3. GBD 2017 Disease and Injury Incidence and Prevalence Collaborators. Global, regional, and national incidence, prevalence, and years lived with disability for 354 diseases and injuries for 195 countries and territories, 1990-2017: a systematic analysis for the Global Burden of Disease Study 2017. Lancet. 2018 Nov 10;392(10159):1789-1858.

4. Ferlay J EM, Lam F, Colombet M, Mery L, Piñeros M, Znaor A, Soerjomataram I, Bray F. Global Cancer Observatory: Cancer Today. Lyon, France: International Agency for Research on Cancer. Published 2018.

5. Khatri M, Malik AS, Bansal M, et al. Effect of supragingival oral irrigation as an adjunct to toothbrushing on plaque accumulation in chronic generalized gingivitis patients. J Indian Soc Periodontol. 2017 Jul-Aug;21(4):296-302.

6. Sen S, Giamberardino LD, Moss K, Morelli T, et al. Periodontal Disease, Regular Dental Care Use, and Incident Ischemic Stroke. Stroke. 2018 Feb;49(2):355-362.

7. Bui FQ, Almeida-da-Silva CLC, Huynh B, Trinh A, Liu J, Woodward J, Asadi $\mathrm{H}$, Ojcius DM. Association between periodontal pathogens and systemic disease. Biomed J. 2019 Feb;42(1):27-35.

8. Băban $A$, Balazsi R, Tăut $D$. Comportamente de sănătate la copiiișiadolescenții din România, Studiu HBSC 2014/2015. Available at: http://insp.gov.ro/sites/1/wp-content/uploads/2014/11/RaportHBSC-Romaniabun.pdf.

9. Funieru C, Twetman S, Funieru E, Dumitrache AM, Sfeatcu RI, Baicus C. Caries experience in schoolchildren in Bucharest, Romania: the PAROGIM study. J Public Health Dent. 2014 Spring;74(2):153-8.

10. Šiljak S, Janković J, Marinković J, Erić M, Janevic T, Janković S. Dental service utilisation among adults in a European developing country: findings from a national health survey. Int Dent J. 2019 Jun; 69(3):200-206.

11. TNS Opinion \& Social at the request of Directorate General Health and Consumers (European Commission). Special Eurobarometer 330. Report oral health. February 2010. Available at: https://ec.europa.eu/ health/non_communicable_diseases/eurobarometers_en.

12. Gurenlian JR. Diagnostic decision making. In: Woodall, IR. Ed. Comprehensive Dental Hygiene Care. 4th ed. St Louis: Mosby; 1993:361-370.

13. Dan AD, Ghergic DL. Knowledge and Skills Level on Oral Health Among Students at the "Ferdinand I" Military Technical Academy in Bucharest. J Med Life. 2020 Oct-Dec;13(4):562-567.

14. Romanian Health Program for Results (P169927). Ministry of Health, Sub-Program for Health Assessment and Promotion and Health Education, 2019:1-21.

15. Cuculescu M, Slusanschi O, Boscaiu VR, et al. Self-reported oral health-related habits, attitudes and knowledge in adults from Portugal, Romania and Sweden - A comparative study. Int J Dent Hyg. 2019 Nov;17(4):359-368. 